\title{
Genome-Editing Technologies in Biomedical Research: The Regulatory Conditions for the Development
}

\author{
Alexander A. Chaplenko, Alexander A. Mokhov, \\ Alexander N. Yavorsky \\ First Moscow State Medical University (Sechenov University); Moscow, Russia \\ Kutafin Moscow State Law University (MSAL); Moscow, Russia \\ Pushchino State Institute of Natural Science; Moscow, Russia
}

\begin{abstract}
Significant progress has been made in the development of genetic technologies in recent decades. Currently, high-performance sequencing and, most importantly, genome editing technologies are widely used and available for laboratories in Russia. Existing technologies are not without drawbacks that significantly hinder further development, in addition, all the necessary reagents and materials, as well as equipment, are produced exclusively abroad. The review highlights the international experience of using genome editing technologies for the treatment and prevention of genetic diseases, vector-borne and viral infections, it offers recommendations for the development of this area in the Russian Federation. Attention is drawn to the legal and ethical regulation, mainly at the level of basic principles. The conclusion is formulated on the need for accelerated adaptation of basic ethical and legal principles for genome editing activities in scientific biomedical activities.
\end{abstract}

Keywords: Genetic technologies, genome editing, CRISPR/Cas, biomedical sciences, regulation, ethics, law

Acknowledgements: Supported by the Ministry of education and science (Topic: Legal regulation accelerated development of genetic technology: scientific and methodological support), No 730000Ф.99.1.БB16AA02001).

Cite as: Chaplenko, A.A., Mokhov, A.A. and Yavorsky A.N., (2021). Genome-Editing Technologies in Biomedical Research: The Regulatory Conditions for the Development. Kutafin Law Review, 8(1), pp. 115128, doi: 10.17803/2313-5395.2021.1.15.115-128 


\section{Contents}

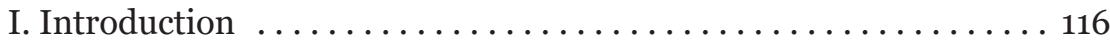

II. Limitations of Modern Genome Editing Techniques . . . . . . 117

III. Development of New Genome Editing Platforms.......... 118

IV. Application of Genome Editing Technologies in Medicine . . . . . 119

V. Human Embryo Genome Editing $\ldots \ldots \ldots \ldots \ldots \ldots \ldots \ldots \ldots \ldots$

VI. The Conditions for the Development ............... 121

VII. Conclusion . . . . . . . . . . . . . . . . . . . . . . . 124

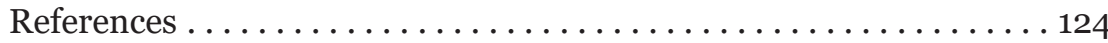

\section{Introduction}

The last decades of intensive development of genetic technologies have been marked by a number of impressive achievements, including the decoding of the complete human genome, the development of the single cell DNA sequencing technology, and, finally, the development of genome editing technologies. Highly efficient genome editing technologies are based on the ability to implement precisely directed double-stranded DNA breaks in the chromosomal region of interest. Numerous non-specific breaks in the DNA occur during the natural process of meiosis, or can be artificially caused by ionizing radiation (Brinkman et al., 2018; Vitelli et al., 2017). Further repair processes can occur by one of two main mechanisms: non-homologous DNA end joining (NHEJ) or homologous recombination (HR). During NHEJ, DNA ends are ligated with minimal enzymatic processing at the endjunction site, while in HR, an intact sister chromatid is usually used as a repair template (Rulten and Grundy, 2017; Wang, Lee and Zha, 2020).

Studies with highly specific genome targeting demonstrated stimulation of both NHEJ and HR in yeast and mammalian cells and, thus a way to programmed genome editing was obtained. Sometimes, errors occur during NHEJ repair, resulting in small local insertions and deletions. These mutations can cause inactivation of the edited gene (Zischewski, Fischer and Bortesi, 2017).

Currently, three powerful classes of nucleases that can be programmed to produce double breaks in essentially any desired target are used in molecular biology: zinc finger nucleases, transcriptional 
activator-like effector nucleases (TAL nucleases), and CRISPR-Cas nucleases. At present, it is CRISPR-Cas that dominates research laboratories around the world, since other methods are less effective, more costly, and laborious (Anzalone, Koblan and Liu, 2020; Germini et al., 2018).

Scientists Emmanuel Charpentier and Jennifer Doudna were awarded the Nobel Prize in Chemistry for 2020 for the development of a genome editing method based on the CRISPR/Cas9 system (The Nobel Prize in Chemistry 2020, 2021).

\section{Limitations of Modern Genome Editing Techniques}

The target of using genome-editing nucleases, in fact, is only producing double-stranded breaks of chromosomal DNA. The main criterion of efficiency is the specificity of the genome-editing platform for a clearly defined region of the genome and the absence of breaks in other loci. However, everything that happens after the rupture is determined by the mechanism of the cellular DNA repair, two variants of which were described earlier. Most somatic cells in higher eukaryotes start the NHEJ process with the concomitant occurrence of insertions and deletions more often than copy sequences from the donor DNA provided. This is acceptable if the purpose of editing is to knock out a gene or a complex of genes, but it significantly limits the possibility of introducing required nucleotide sequences. In studies (Karagyaur, Rubtsov, Vasiliev and Tkachuk, 2018; Paix et al., 2017), limited success has been achieved in modulating the ratio between the target and the mutant product, but so far no universal solution has been found, and for some types of cells, NHEJ remains the most frequently occurring repair method. Several recent publications report that small molecule inhibitors of key enzymes in the NHEJ process can be effective, but more research is needed to create more reliable reagents (Bischoff, Wimberger, Maresca and Brakebusch, 2020; Li et al., 2017). Another way of increasing the efficiency of insertion of the target gene is the modification of the donor DNA molecule, linking the donor sequence with the guide RNA, and using natural mechanisms for inserting the 
desired fragments (Chen and Knoepfler, 2016; Chuai et al., 2018; Dyikanov et al., 2019; Long et al., 2018).

On the other hand, all DNA editing platforms have high but limited specificity. One of the latest studies has shown the possibility of increasing the specificity of CRISPR-Cas by modulating the Cas9 protein and guiding RNA. It depends on the application how important is the absolute specificity of the editing system, as well as the absence of mutagenic potential. In many model organisms, there are ways to prevent the expression of a mutant gene, for example, by knocking it out and replacing it with a wild-type genome. We can rely on these mechanisms when editing the genome of plant or bacterial cells, as well as when creating humanized animal models for pharmacological research (Hackett et al., 2018; Hua, Wang, Huang and Wang, 2017). Even in some medical applications, off-target mutations may be acceptable if they do not lead to a disease, but this aspect is the most ethically vulnerable (Brokowski and Adli, 2019).

According to the established bioethical literature, international and national ethical (bioethical) principles, the doctor and the researcher should always be guided by the principle of non-harm, and the degree of the existing risk should not exceed the existing problem (real, not imaginary). According to the Code of Professional Ethics of a Doctor of the Russian Federation (adopted by the National Congress of Doctors on October 5,2012 ), a doctor engaged in scientific activities should not use his scientific knowledge to the detriment of the health and safety of the patient or society. The priority of the patient's interests is established as a principle and in Art. 4 of the Federal Law No 323-FZ dated 21.11.2011 "On the fundamentals of the public health protection in the Russian Federation".

\section{Development of New Genome Editing Platforms}

Genome editing will probably remain a widely used tool in both scientific research and commercial and medical fields. However, there arises a question: is CRISPR-Cas the last word in programmable nucleases, or perhaps there is something even better on the horizon. At the moment, it is difficult to imagine a system that is significantly 
simpler than recognition of a gene by a complementary matrix and cleavage by a single protein. It is possible to develop a chemical system based on low molecular weight synthetic compounds that combine DNA recognition with its cleavage. Research aimed at achieving this goal has been going on for decades - from triplex-forming oligonucleotides to peptide nucleic acids and polyimines (Eid, Alshareef and Mahfouz, 2018; Koonin, Makarova and Zhang, 2017; Lee et al., 2018), but the development of the platform with adequate specificity and efficiency is still far away. It seems that new methods of genome editing will be discovered through research into natural processes rather than through optimization of CRISPR techniques. A variant of gaps-induced genome editing is CRISPR-mediated base editing. This technology uses nickase Cas9, which edits only one strand of the target DNA. Conversion of cytisine to uracil within a few base pairs closest to the RNA binding site results in changes of expression in this very narrow region. Future uses of this approach may include modification of individual alleles of human genes.

\section{Application of Genome Editing Technologies in Medicine}

At the moment, a large number of attempts to use genome editing technologies in clinical practice have been described. FDA has approved a large number of clinical studies involving somatic cell genome editing for phase I clinical trials. The earliest studies used zinc finger nucleases to knock out the $\mathrm{CCR}_{5}$ receptor gene in $\mathrm{T}$ lymphocytes of HIV-positive patients (Liu et al., 2017), this modification makes T cells resistant to the virus. In the future, it is planned to edit the genome of lymphocyte progenitor cells and even individual cells of the human embryo (Cyranoski, 2019).

TAL nucleases have been used to enhance the efficacy of CAR-T cell therapy (Lucibello, Menegatti and Menger, 2020); in addition, two studies using CRISPR/Cas9 have been approved for this purpose. These examples are based on genome editing of cells previously isolated from the body, followed by administration to the same patient from whom the collection was made (autologous biomedical cell products). 
Such ex vivo procedures allow for easy delivery of editing systems into cells, as well as the ability and preliminary characterization of edited cells. During the development of cell therapy methods, genome editing will become an integral complement to them. In many cases, cell therapy is not possible (for example, it is impossible to isolate all or even most of the target cells). Currently, clinical trials of agents for the treatment of hemophilia and lysosomal storage diseases are underway, based on the delivery of zinc finger nucleases in vivo by viral vectors. Thus, the genome of hepatocytes, which are classified as the type of cells that are readily available for introduction, is edited. Delivery into other organs in vivo will require the creation of new vector and non-vector approaches and, possibly, the creation of specific lines of genetically modified stem cells. Active research is directed towards the treatment of other genetic diseases, including sickle cell anemia and muscular dystrophy. As with any medical application, genomic editing systems must be proved to be effective and safe (Gerace et al., 2017; Kick, Kirchner and Schneider, 2017; You et al., 2019).

\section{Human Embryo Genome Editing}

Due to the ease of editing the genome using the CRISPR platform and, accordingly, the wide potential for abuse of the technology, there is a considerable interest in the prospects for editing the genome of the human embryo. The main method of application is the delivery of editing agents into the cells of an embryo created by in vitro fertilization. In the future, it may be more appropriate and ethically acceptable to edit gametogenic progenitor cells in the future parents. The advantage of embryonic correction of gene alleles corresponding to pathological conditions is that they will disappear from the genome forever. However, there is a risk that trying to correct the genetic code of an unborn child could do more harm than good. Modern genome editing technology does not have sufficient efficiency and specificity to fully guarantee safety. Mutations arising at off-target chromosome loci as a result of the introduction of editing constructs can affect the child's body and be transmitted from generation to generation, and their effects will not always be benign, predictable or reversible. 
The world's first operation to edit the genome of a human embryo was performed in 2018 in China. Chinese geneticist He Jiankui performed in vitro fertilization and then edited the genomes of the resulting embryos using CRISPR/Cas9 technology, creating an artificial mutation in the CCR5- $\Delta 32$ gene, which should provide future children with immunity to the human immunodeficiency virus. As a result, the first twin girls in the history of mankind were born with an edited genome ("The CRISPR-baby") (Greely, 2019).

Ongoing research will make embryonic genome editing safer and more efficient, and it seems inevitable that it will eventually be widely used. At the same time, it is important to discuss both legal (Mokhov, Levushkin and Yavorsky, 2020) and ethical (Cribbs and Perera, 2017) issues related to the editing of the human embryo genome.

\section{The Conditions for the Development}

During the development of molecular biology and biomedicine, genome editing technologies are being improved and developed, their safety and effectiveness are increasing. The main leaders in this area of research are China, the United States and the EU. Russian scientists are also actively involved in global genetic research. According to the level of qualification, Russian researchers are comparable to employees of the world's leading genetic laboratories, which allows not only to effectively use, but also to develop modern genome editing technologies (Rebrikov, 2021). However, to date there is a significant lag in the country's production of its own equipment, reagents, materials, software and digital databases necessary for the introduction of the developed genetic technologies in industrial production and medical practice in Russia.

In the modern world, the ability to have their own genetic technologies and molecular platforms for genome editing is certainly necessary both for the development of bioeconomics and for ensuring the biosafety of the state. Recently, the Russian Federation has taken a number of strategically important steps to accelerate the development of genetic technologies to achieve the goals of the national bioeconomy and biosafety of the country's population. The legal basis for the accelerated 
development of this scientific and technical direction in the country was a number of policy documents: the Decree of the President of the Russian Federation dated 28.11.2018 No 680 "About the development of genetic technologies in the Russian Federation", the Resolution of the Government of the Russian Federation dated 22.04.2019 No 479 "On the approval of the Federal scientific and technical program of development of genetic technology in the years 2019-2027", and the Order of the Government of the Russian Federation dated 26.10.2019 No 2535-R "On the approval of the list of organizations on the basis of which world class genomic research centers are established".

An important practical step towards the implementation of these documents was the signing in 2020 of an Agreement on Cooperation between the Government of the Russian Federation and Public Joint Stock Company "Rosneft Oil Company" in order to solve the problems of accelerated development of genetic technologies in the Russian Federation and the country's entry into the world's leading positions in this field of science and technology.

One of the important conditions for solving these strategic state tasks is the creation of a comfortable regulatory environment for the development of national genetic technologies, in the form of improving the regulatory framework and eliminating administrative and technical barriers. A special working group of experts has been established under the Presidium of the Council for the Implementation of the Federal Scientific and Technical Program for the Development of Genetic Technologies for 2019-2027 to coordinate the development of legal regulation in the field of genetic technologies, including genomic editing. Under the auspices of the working group, within the framework of the state task of the Ministry of Science and Higher Education of the Russian Federation, expert and analytical studies on the topic "Legal regulation of accelerated development of genetic technologies: scientific and methodological support" are being conducted at the Kutafin Moscow State Law University.

${ }^{1}$ Order of the Government of the Russian Federation dated 02.03.2020 No 481-r. 
Another prerequisite is the launch of an import substitution program aimed at reducing the dependence of the development of national genetic technologies on foreign equipment, reagents, materials, software and digital databases.

Against the background of solving a number of scientific and technical tasks, first of all it is necessary to ensure the priority of the humanistic approach at the stage of solving questions about the possibility of practical application of genome editing technologies in medicine, since the introduction of any new medical technology potentially entails risks for the health and life of patients. Existing international law and relevant national legislation clearly classify the preservation of the genome as a fundamental human right. ${ }^{2}$

In terms of the level of knowledge intensity and the speed of development, genetic editing technologies are quite comparable to the most advanced digital technologies, which makes it possible to combine them with the general concept of "High Technologies of the 21st Century". A common characteristic of these high technologies is the situation of a large time gap between the actual beginning of their practical use and the formation of the legislative and regulatory framework governing the process of their official introduction and application. In the context of these realities of the 21st century, it seems reasonable to form and develop a new direction of humanitarian science - the ethics of high technologies. In the system of the Ministry of Health of the Russian Federation, there is an Ethical Council that conducts ethical expertise when deciding on the possibility of conducting clinical trials of new drugs in humans (Khokhlov, Chudova and Tsyzman, 2018). By analogy with this traditional approach for medicine, the idea of creating a specialized collegial expert body to consider the possibility of conducting the first clinical studies of genetic technologies in humans should be considered (Mokhov and Yavorsky, 2020).

2 The Universal Declaration on the Human Genome and Human Rights (adopted on 11.11.1997 by the General Assembly of the United Nations). Available at: https:// www.ohchr.org/EN/ProfessionalInterest/Pages/HumanGenomeAndHumanRights. aspx [Accessed 10.07.2020]. 


\section{Conclusion}

In general, the new state policy of the Russian Federation in the field of development of national genetic technologies is aimed at a significant increase in funding and rapid deployment of promising scientific and technological programs at rapid achievement of practical results, the creation of real technologies for high-precision genome editing, and the production of competitive biotechnological products both in Russia and in the world. A striking example of the successful implementation of the new policy of development of national genetic technologies was the fact of using genome editing technology to create the first Gam-COVIDVac vaccine registered in the world at the National Research Center of Epidemiology and Microbiology named after Honorary Academician N.F. Gamalei (Logunov et al., 2020).

\section{References}

1. Anzalone, A., Koblan, L. and Liu, D., (2020). Genome editing with CRISPR-Cas nucleases, base editors, transposases and prime editors. Nature Biotechnology, 38(7), pp. 824-844.

2. Bischoff, N., Wimberger, S., Maresca, M. and Brakebusch, C., (2020). Improving Precise CRISPR Genome Editing by Small Molecules: Is there a Magic Potion? Cells, 9(5), p. 1318.

3. Brinkman, E., Chen, T., de Haas, M., Holland, H., Akhtar, W. and van Steensel, B., (2018). Kinetics and Fidelity of the Repair of Cas9Induced Double-Strand DNA Breaks. Molecular Cell, 70(5), pp. 801813.e6.

4. Brokowski, C. and Adli, M., (2019). CRISPR Ethics: Moral Considerations for Applications of a Powerful Tool. Journal of Molecular Biology, 431(1), pp. 88-101.

5. Chen, K. and Knoepfler, P., (2016). To CRISPR and beyond: the evolution of genome editing in stem cells. Regenerative Medicine, 11(8), pp. 801-816.

6. Chuai, G., Ma, H., Yan, J., Chen, M., Hong, N., Xue, D., Zhou, C., Zhu, C., Chen, K., Duan, B., Gu, F., Qu, S., Huang, D., Wei, J. and Liu, Q., (2018). DeepCRISPR: optimized CRISPR guide RNA design by deep learning. Genome Biology, 19(1). 
7. Cribbs, A. and Perera, S., (2017). Science and bioethics of CRISPRCAS9 gene editing: An analysis towards separating facts and fiction. Yale Journal of Biology and Medicine, 9o(4), pp. 625-634.

8. Cyranoski, D., (2019). Russian 'CRISPR-baby' scientist has started editing genes in human eggs with goal of altering deaf gene. Nature, 574(7779), pp. 465-466.

9. Dyikanov, D., Vasiluev, P., Rysenkova, K., Aleksandrushkina, N., Tyurin-Kuzmin, P., Kulebyakin, K., Rubtsov, Y., Shmakova, A., Evseeva, M., Balatskiy, A., Semina, E., Rostovtseva, A., Makarevich, P. and Karagyaur, M., (2019). Optimization of CRISPR/Cas9 Technology to Knock Out Genes of Interest in Aneuploid Cell Lines. Tissue Engineering . Part C: Methods, 25(3), pp. 168-175.

10. Eid, A., Alshareef, S. and Mahfouz, M., (2018). CRISPR base editors: genome editing without double-stranded breaks. Biochemical Journal, 475(11), pp. 1955-1964.

11. Gerace, D., Martiniello-Wilks, R., Nassif, N., Lal, S., Steptoe, R. and Simpson, A., (2017). CRISPR-targeted genome editing of mesenchymal stem cell-derived therapies for type 1 diabetes: a path to clinical success? Stem Cell Research \& Therapy, 8(1).

12. Germini, D., Tsfasman, T., Zakharova, V., Sjakste, N., Lipinski, M. and Vassetzky, Y., (2018). A Comparison of Techniques to Evaluate the Effectiveness of Genome Editing. Trends in Biotechnology, 36(2), pp. 147-159.

13. Greely, H., (2019). CRISPR'd babies: human germline genome editing in the 'He Jiankui affair'. Journal of Law and the Biosciences, 6(1), pp. 111-183.

14. Hackett, J., Huang, Y., Günesdogan, U., Gretarsson, K., Kobayashi, T. and Surani, M., (2018). Tracing the transitions from pluripotency to germ cell fate with CRISPR screening. Nature Communications, 9(1).

15. Hua, Y., Wang, C., Huang, J. and Wang, K., (2017). A simple and efficient method for CRISPR/Cas9-induced mutant screening. Journal of Genetics and Genomics, 44(4), pp. 207-213.

16. Karagyaur, M., Rubtsov, Y., Vasiliev, P. and Tkachuk, V., (2018). Practical Recommendations for Improving Efficiency and Accuracy of the CRISPR/Cas9 Genome Editing System. Biochemistry (Moscow), 83(6), pp. 629-642. 
17. Khokhlov, A., Chudova, N. and Tsyzman, L., (2018). The results of the analysis of the expert work of the Council on Ethics of the Ministry of Health of the Russian Federation. Meditsinskaya Etika, 2, pp. 17-22. (In Russ.).

18. Kick, L., Kirchner, M. and Schneider, S., (2017). CRISPR-Cas9: From a bacterial immune system to genome-edited human cells in clinical trials. Bioengineered, 8(3), pp. 280-286.

19. Koonin, E., Makarova, K. and Zhang, F., (2017). Diversity, classification and evolution of CRISPR-Cas systems. Current Opinion in Microbiology, 37, pp. 67-78.

20. Lee, J., Jeong, E., Lee, J., Jung, M., Shin, E., Kim, Y., Lee, K., Jung, I., Kim, D., Kim, S. and Kim, J., (2018). Directed evolution of CRISPR-Cas9 to increase its specificity. Nature Communications, 9(1).

21. Li, G., Zhang, X., Zhong, C., Mo, J., Quan, R., Yang, J., Liu, D., Li, Z., Yang, H. and Wu, Z., (2017). Small molecules enhance CRISPR/ Cas9-mediated homology-directed genome editing in primary cells. Scientific Reports, 7(1).

22. Liu, Z., Chen, S., Jin, X., Wang, Q., Yang, K., Li, C., Xiao, Q., Hou, P., Liu, S., Wu, S., Hou, W., Xiong, Y., Kong, C., Zhao, X., Wu, L., Li, C., Sun, G. and Guo, D., (2017). Genome editing of the HIV coreceptors CCR 5 and CXCR4 by CRISPR-Cas9 protects CD4+ T cells from HIV-1 infection. Cell \& Bioscience, 7(1).

23. Logunov, D., Dolzhikova, I., Zubkova, O., Tukhvatulin, A., Shcheblyakov, D., Dzharullaeva, A., Grousova, D., Erokhova, A., Kovyrshina, A., Botikov, A., Izhaeva, F., Popova, O., Ozharovskaya, T., Esmagambetov, I., Favorskaya, I., Zrelkin, D., Voronina, D., Shcherbinin, D., Semikhin, A., Simakova, Y., Tokarskaya, E., Lubenets, N., Egorova, D., Shmarov, M., Nikitenko, N., Morozova, L., Smolyarchuk, E., Kryukov, E., Babira, V., Borisevich, S., Naroditsky, B. and Gintsburg, A., (2020). Safety and immunogenicity of an rAd26 and rAd5 vector-based heterologous prime-boost COVID-19 vaccine in two formulations: two open, non-randomised phase 1/2 studies from Russia. The Lancet, 396(10255), pp. 887-897.

24. Long, L., Guo, D., Gao, W., Yang, W., Hou, L., Ma, X., Miao, Y., Botella, J. and Song, C., (2018). Optimization of CRISPR/Cas9 genome editing in cotton by improved sgRNA expression. Plant Methods, 14(1).

25. Lucibello, F., Menegatti, S. and Menger, L., (2020). Methods to edit T cells for cancer immunotherapy. Methods in Enzymology, 631, pp. 107-135. 
26. Mokhov, A., Levushkin, A. and Yavorsky, A., (2020). Genome Editing of Human Embryo: Allow or Prohibit? Journal of Advanced Research in Law and Economics, 11(2), p. 483. (In Russ.).

27. Mokhov, A. and Yavorsky, A., (2020). High-Tech Ethics: Establishment Issues and Development Prospects. Jurist, 12, pp. 2-8. (In Russ.).

28. NobelPrize.org. 2021. The Nobel Prize in Chemistry 2020. [online] Available at: https://www.nobelprize.org/prizes/chemistry/2020/ press-release [Accessed 22.03. 2021].

29. Paix, A., Folkmann, A., Goldman, D., Kulaga, H., Grzelak, M., Rasoloson, D., Paidemarry, S., Green, R., Reed, R. and Seydoux, G., (2017). Precision genome editing using synthesis-dependent repair of Cas9-induced DNA breaks. Proceedings of the National Academy of Sciences, 114(50), pp. E10745-E10754.

30. Rebrikov, D., (2021). Human genome editing. Bulletin of RSMU, 3, pp. 4-14.

31. Rulten, S. and Grundy, G., (2017). Non-homologous end joining: Common interaction sites and exchange of multiple factors in the DNA repair process. BioEssays, 39(3), p. 1600209.

32. Vitelli, V., Galbiati, A., Iannelli, F., Pessina, F., Sharma, S. and d'Adda di Fagagna, F., (2017). Recent Advancements in DNA DamageTranscription Crosstalk and High-Resolution Mapping of DNA Breaks. Annual Review of Genomics and Human Genetics, 18(1), pp. 87-113.

33. Wang, X., Lee, B. and Zha, S., (2020). The recent advances in non-homologous end-joining through the lens of lymphocyte development. DNA Repair, 94, p. 102874.

34. You, L., Tong, R., Li, M., Liu, Y., Xue, J. and Lu, Y., (2019). Advancements and Obstacles ofCRISPR-Cas9 Technology in Translational Research. Molecular Therapy - Methods \& Clinical Development, 13, pp. 359-370.

35. Zischewski, J., Fischer, R. and Bortesi, L., (2017). Detection of on-target and off-target mutations generated by CRISPR/Cas9 and other sequence-specific nucleases. Biotechnology Advances, 35(1), pp. 95104. 


\section{Information about the authors}

Alexander A. Chaplenko, Cand. Sci., Associate Professor, Department of Organization and Management in the Field of Drugs Circulation, First Moscow State Medical University (Sechenov University)

Bolshaya Pirogovskaya St., 2, build. 4, Moscow, 119435

a.a.chaplenko@yandex.ru

Alexander A. Mokhov, Dr. Sci. (Law), Professor, Head of the Department of Medical Law, Kutafin Moscow State Law University (MSAL)

Sadovaya-Kudrinskaya St., 9, Moscow, Russia, 125993

aamohov@msal.ru

Alexander N. Yavorsky, Dr. Sci. (Medicine), Professor, Chief Researcher, Pushchino State Institute of Natural Science

Nauki Av., 3, Pushchino, Moscow Region, 142290

200-31-11@mail.ru 